\title{
Infrared Camera-based Detection and Analysis of Barrels in Rotary Kilns for Waste Incineration
}

\author{
by P. Waibel*, M. Vogelbacher*, J. Matthes* and H.B. Keller*
}

^Institute for Applied Computer Science, Karlsruhe Institute of Technology, Germany, patrick.waibel@kit.edu

\begin{abstract}
Rotary kilns are widely used for the thermal treatment of highly toxic waste. The waste (mostly organic compounds) is packed in barrels before it is fed in the kiln. Depending on the compound the heating inside the kiln leads to deflagrations of the barrels associated with an incomplete burnout. In this paper we present a new method to detect and track waste barrels in infrared image sequences of rotary kilns for waste incineration online. Besides a better insight in the process behaviour this method provides the possibility of fast interventions of the process control e.g. in order to circumvent carbon monoxide peaks in the exhaust emission.
\end{abstract}

\section{Introduction}

Chemical industry production is often attended by the emergence of hazardous waste. Due to its toxicity this waste is often inappropriate for a direct landfill. A thermal treatment is required to transform it in less dangerous substances. Therefore temperatures inside a kiln around $600-1000^{\circ} \mathrm{C}$ are necessary. A rotary kiln is a cylindrical vessel that is slightly inclined to the horizontal.

A particular challenge for the image processing system are the various process conditions inside the kiln as well as the particle load in the gas phase which leads to disturbances in the acquired images. Additionally, the low image resolution and cakings at the kiln end influence the image processing. [1]

\section{Image Acquisition}

A custom-built infrared camera system with air supply and water cooling was installed at the lower end of the kiln (Figure 1). The camera position is slight on the top-right of the kiln's rotary axis.

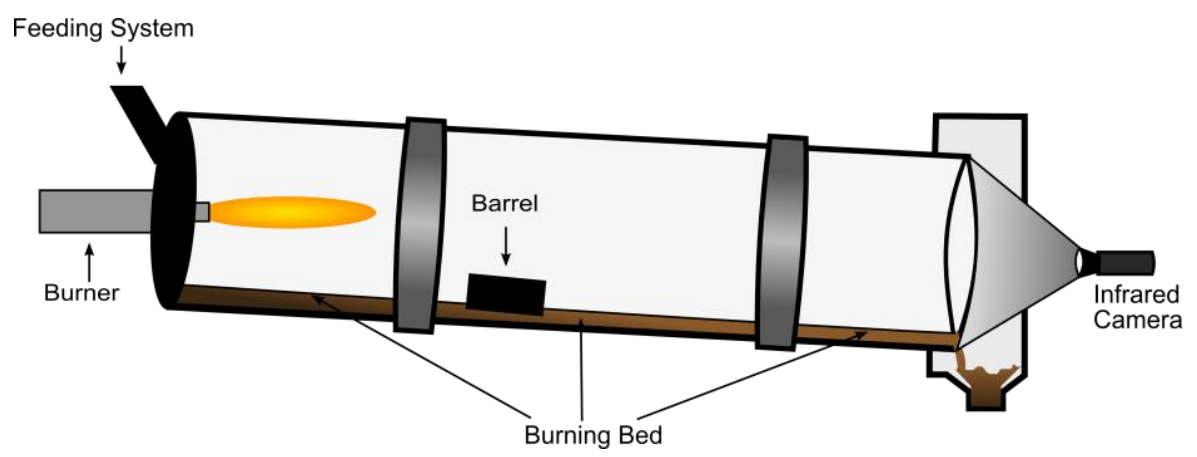

Fig. 1. Rotary kiln for waste incineration with infrared camera

The camera is sensitive at wavelength of 3-5 $\mu \mathrm{m}$. A spectral band pass filter at $3.9 \pm 0.1 \mu \mathrm{m}$ allows for a high transmission in the gas phase, since relevant flue gas molecules have a local minimum of absorption at this wavelength. Figure 2 shows a simulation of spectral transmissivities inside a typical kiln atmosphere at a temperature of $1000{ }^{\circ} \mathrm{C}$ in a $5 \mathrm{~m}$-distance. The image resolution is $320 \times 240$ pixels and the images were acquired with $50 \mathrm{fps}$. The image pixel values reflect absolute temperatures. The available image data was acquired during a measuring campaign of two days. [2]

The analyzed kiln is $5 \mathrm{~m}$ long with a radius of $1.5 \mathrm{~m}$ and a rotational velocity of about $1 \mathrm{rpm}$. The plant's capacity is about $75.000 \mathrm{t} / \mathrm{a}$. Besides the kiln feeding system it comprises a liquid burner as well as a burner for paste-like materials. Barrels are inserted into the rotary kiln via a feeding system. After being thermally converted the solid compounds are discharged in a slag remover at the lower end of the kiln. The flue gas is led to an afterburner chamber to ensure a complete burnout. In a heat recovery system the flue gas is used to generate process steam before it is cleaned in a multi-stage cleaning system. The layout of a hazardous waste incineration plant is depicted in figure 3. Further information on the kiln can be found in [3]. 


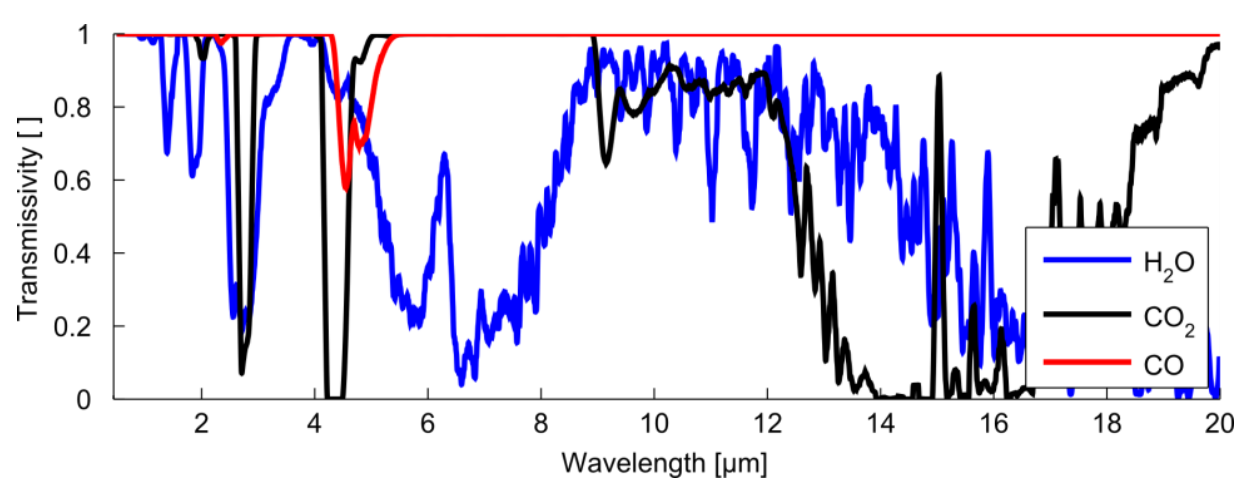

Fig. 2. Transmissivities of relevant flue gas molecules $\left(\mathrm{H}_{2} \mathrm{O}(250 \mathrm{~g} / \mathrm{kg}), \mathrm{CO}_{2}(200 \mathrm{~g} / \mathrm{kg}), \mathrm{CO}(30 \mathrm{~g} / \mathrm{kg})\right)$

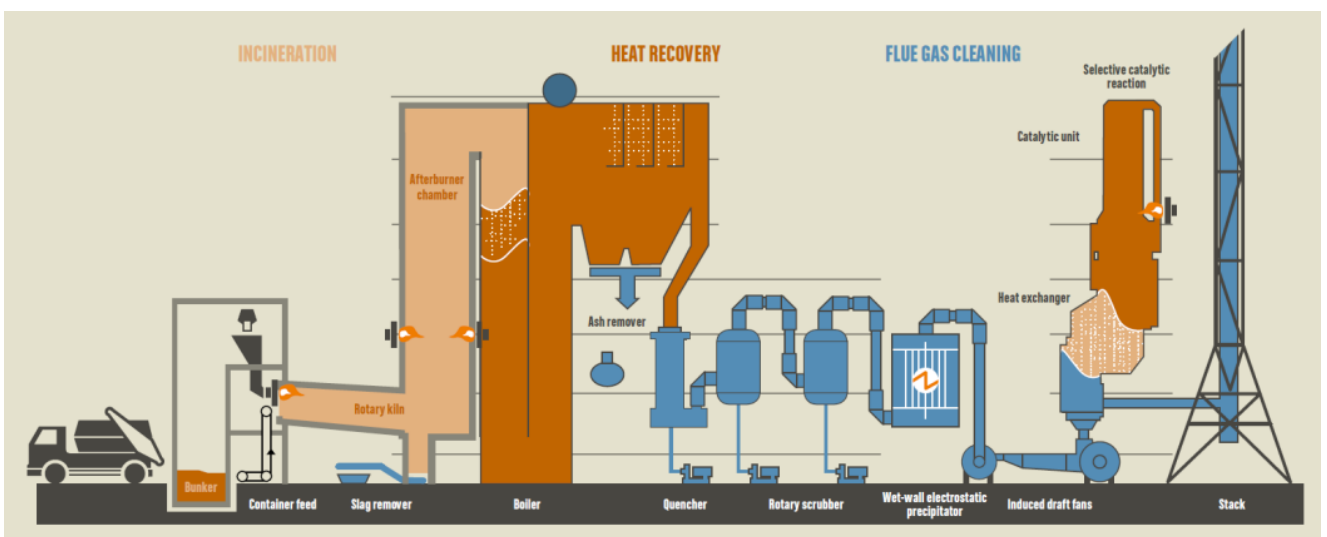

Fig. 3. Scheme of a hazardous waste incineration plant [3]

\section{Image Processing}

The new developed image processing algorithms provide the possibility to detect barrels in the acquired images and extract features such as their positions or sizes. Figure 4 shows a typical image and an explanation of the relevant image regions. The new developed algorithm including its single steps is outlined in figure 5.

\subsection{Image Pre-processing}

The first step of the image processing system is the determination of the region of interest (ROI). The ROI can coarsely be defined manually by constructional restrictions of the kiln. Using dynamic as well as intensity-based features the caking at the kiln end can be detected and reduced from the manually predefined ROI. An algorithm to detect high soot loads in the gas phase is implemented in order to block further calculations when the image quality is too low.
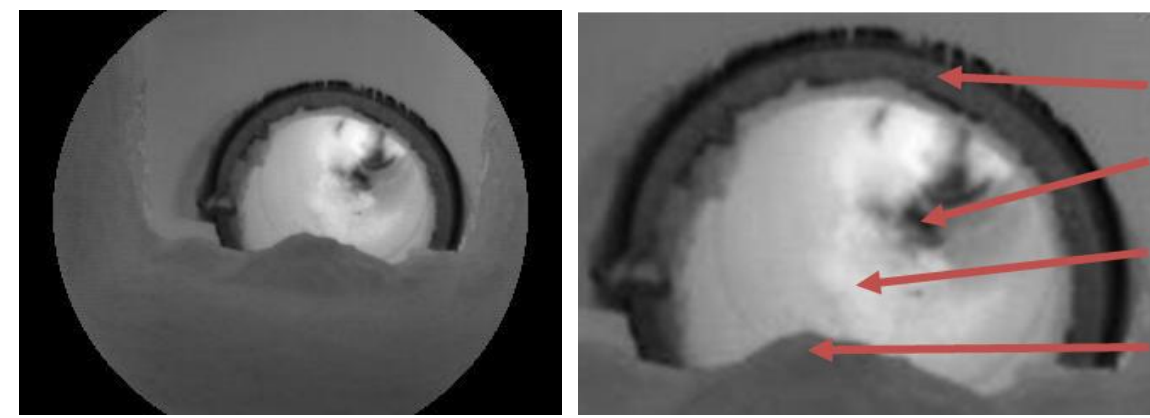

Rotary Kiln

Barrel

Burning

Bed

Caking

Fig. 4. Original image (left) and cropped image with identifiers (right) 


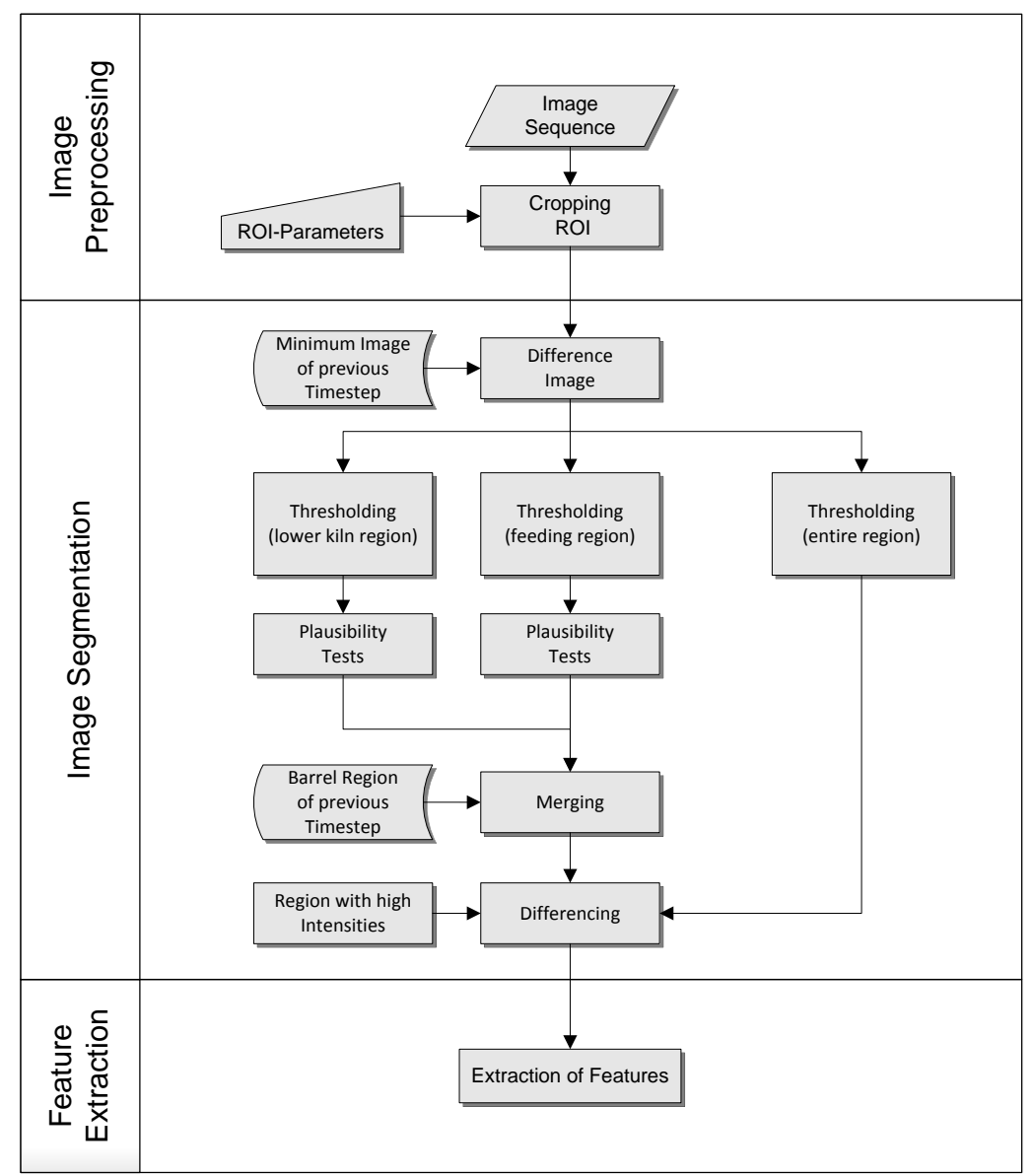

Fig. 5. Image processing algorithm

\subsection{Segmentation}

In the segmentation process the barrels are discriminated from the image background. The new developed segmentation method makes use of dynamic properties via difference images and temporal filters as well as intensity properties via temperature differences. Prior domain knowledge is included in the algorithm to further improve robustness.

Flame fluctuations in the burning bed can cause a temporary coverage of barrels in the images, which leads to erroneous detections. Due to a moving minimum filtering of the images of length $\mathrm{N}_{\min }$ the influence of these interfering fluctuations at high temperatures can be reduced. Cold image regions such as barrels are emphasized in the resulting image $g_{\min }$.

$$
g_{\min }\left(x, y, t_{k}\right)=\min \left\{g\left(x, y, t_{k}-N_{\min }\right), \ldots, g\left(x, y, t_{k}\right)\right\}
$$

Concerning the heavily varying conditions (temporal as well as spatial) a solely intensity-based segmentation is not suitable for a robust and reliable segmentation. Therefore dynamic properties of the barrels are addressed. On the basis of difference images $g_{\text {diff }}$ of two subsequent images the pixel values are regarded.

$$
g_{\text {diff }}\left(x, y, t_{k}\right)=g_{\text {min }}\left(x, y, t_{k}\right)-g_{\text {min }}\left(x, y, t_{k-1}\right)
$$

Negative values point to a change from hot to cold (index: h2c). This can be found at pixels where a barrel is located for the first time, e.g. directly after being fed in the kiln or because of a movement of the barrel inside the kiln. Positive values indicate a movement of the barrel (index: $\mathrm{c} 2 \mathrm{~h}$ ). In this case a pixel turns from being associated with the barrel region back to background (kiln wall, burning bed). The segmentation based on the difference images is performed by thresholding. 
Spatial dependant threshold values can be defined to raise the robustness. It appeared that two threshold values, a high one $\left(s_{1}\right)$ for the area near the feeding system and a lower one $\left(s_{2}\right)$ for the rest performed well for the acquired image sequence. Particularly near the feeding area long-term flame fluctuations are presenting themselves problematic. By means of the higher threshold value false segmentations can be reduced. Both regions are unified after thresholding.

$$
\begin{aligned}
& \Omega_{h 2 c}\left(t_{k}\right)=\left\{x, y \in \Omega_{R O I} \mid g_{\text {diff }}\left(x, y, t_{k}\right)<s_{1 / 2}\right\} \\
& \Omega_{c 2 h}\left(t_{k}\right)=\left\{x, y \in \Omega_{R O I} \mid g_{\text {diff }}\left(x, y, t_{k}\right)>s_{3}\right\}
\end{aligned}
$$

Further plausibility criteria can be integrated to ensure the segmentation. On the one hand very small connected regions can be disregarded. On the other hand the intensity value can be used as criterion. Depending on the mean temperature in the kiln a threshold value can be defined below which a segmented pixel's intensity has to be for getting confirmed.

The found region $\Omega_{\mathrm{h} 2 \mathrm{c}}$ has to be unified with the region of the last time step in order to circumvent that solely pixels with high negative difference values in the current time step are accounted for. Pixels with high difference values in the current time step $\left(\Omega_{\mathrm{c} 2 \mathrm{~h}}\right)$ have to be removed from the segmentation result due to the movement of the barrel.

$$
\Omega_{\text {Barrel }}\left(t_{k}\right)=\left(\Omega_{h 2 c}\left(t_{k}\right) \cup \Omega_{\text {Barrel }}\left(t_{k-1}\right)\right) / \Omega_{c 2 h}\left(t_{k}\right)
$$

Again, the resulting region can be plausibilized with an adaptive intensity threshold value. The final segmentation result can be used for the feature extraction. In figure 6 an image sequence with a segmented barrel is shown.

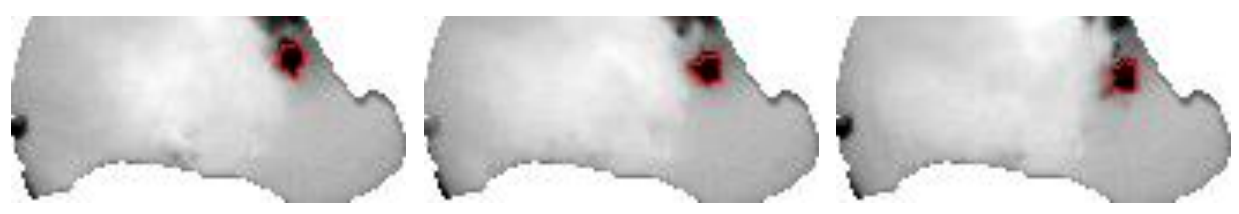

Fig. 6. Image sequence with segmented barrel

\subsection{Feature Extraction}

\subsubsection{Size of the barrel}

A significant feature that can be extracted is the size of the segmented barrel. An abrupt size reduction can be regarded as the consequence of a higher flame occurrence in the region of the barrel. Therefore it can be deduced that the burnout of the barrel has started and a higher soot load is expected soon after. In order to analyze this feature concerning relevance and robustness the average difference values in the upper image part displaying the lower afterburner chamber are taken into account. As shown in figure 7 and figure 8 deflagrations cause high difference values in the upper part of the image. Nevertheless the mean difference in this region cannot be used as an early indicator since a deflagration is noticed too late to intervene with the process control in time.

Figure 9 to figure 11 depict the barrel size compared to the mean intensity of the difference images based on three different image sequences. The increasing mean intensity follows from the rising emissions caused by deflagrations of the barrel. 

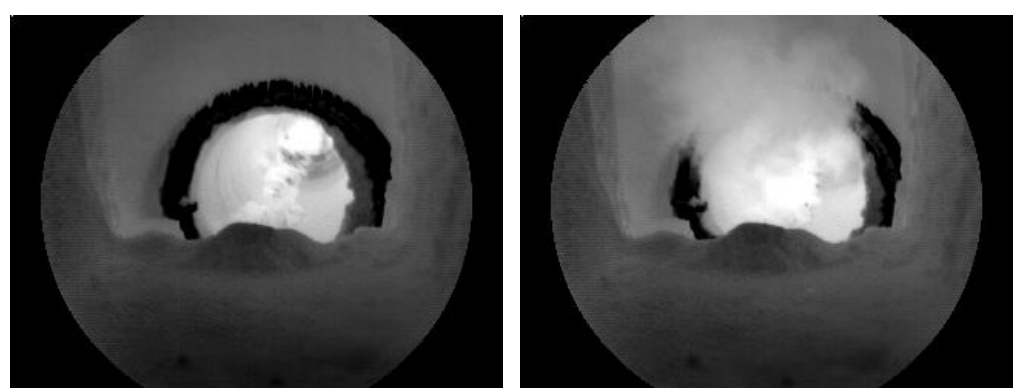

Fig. 7. Original Images (left: without deflagration, right: with deflagration)

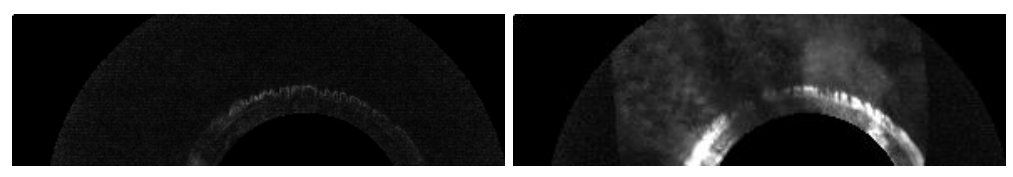

Fig. 8. Difference Images (left: without deflagration, right: with deflagration)

In sequence 1 (figure 9) an increase of the barrel's size at 14:53 is followed by a decrease shortly after. The change happens before the mean intensity of the difference images increases. This can be explained by the ignition of the barrel with a rise of the temperature in the barrel's region. Sequence 2 (figure 10) shows a similar behaviour. An increase and decrease of the barrel's size can be observed at 13:22. Here also a deflagration takes place subsequently. Nevertheless sequence 3 (Figure 11) shows a deviant behaviour. After the reduction of the barrel's size there is no shortterm deflagration but a long-term burning of the barrel which leads to a longer variation of the intensity values. This is due to the varying contents of the barrels. If the heating value is low a slow burnout instead of a deflagration takes place.
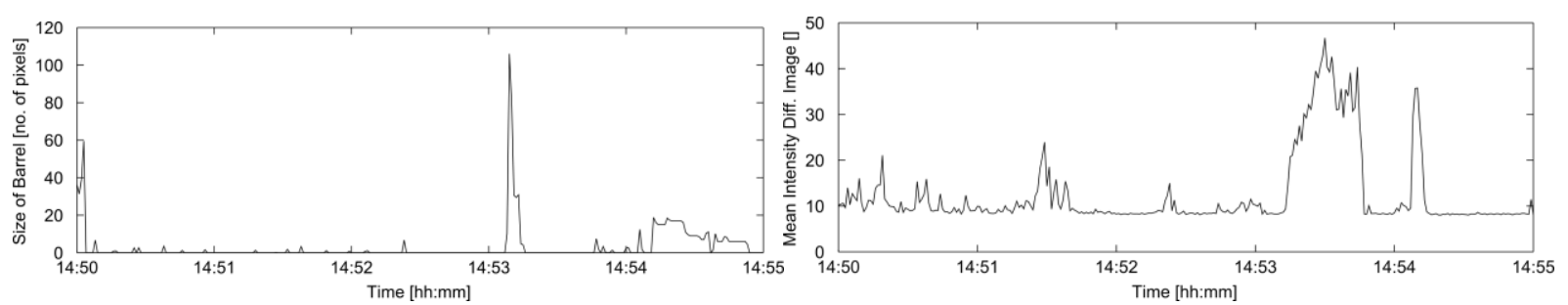

Fig. 9. Sequence 1 - Size of segmented barrel (left); mean intensity of difference image (right)
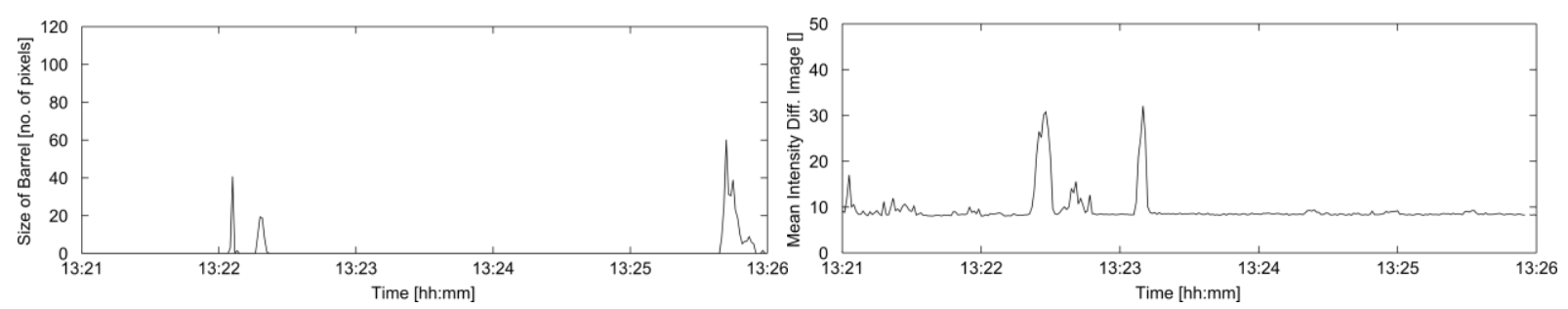

Fig. 10. Sequence 2 - Size of segmented barrel (left); mean intensity of difference image (right)
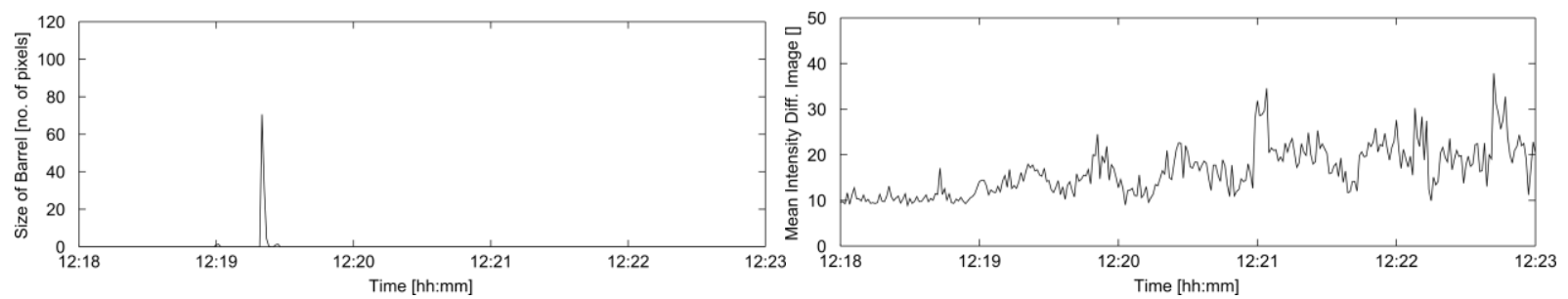

Fig. 11. Sequence 3 - Size of segmented barrel (left); mean intensity of difference image (right) 
However the barrel's size can be used as an early detector of a deflagration if the barrel's heating value is known beforehand. The process control could use the feature barrel's size to adapt its actuating variables accordingly. One possibility is e.g. to accurately time the increasing of the oxygen fraction in the afterburner chamber to avoid carbon monoxide peaks in the exhaust emission. To improve the accuracy of the counter-measures it could be convenient to integrate information on the barrel's contents.

\subsubsection{Vertical position of the barrel}

The maximum vertical position of the barrel in the images is another significant feature which can be extracted from the segmentation result. It indicates the depth position of the barrel in the kiln and allows for the detection of barrels rolling through the whole kiln with just a partial burnout respectively without having ignited yet. Such process situations are undesired because the slag has to be fed into the kiln again due to an incomplete conversion of the toxic materials. In Figure 12 an image sequence is shown where a barrel (low energetic content) is observable which is almost rolling through the whole kiln, before it stops and its content is pouring out. The extracted maximum vertical position is depicted in figure 13. The barrel is detected shortly after 14:31 and then fast moves to the end of the kiln where it stops. Between 14:32 and 14:33 the burning starts and the barrel cannot be detected any longer. The dropping during the detection is caused by an incorrect segmentation.
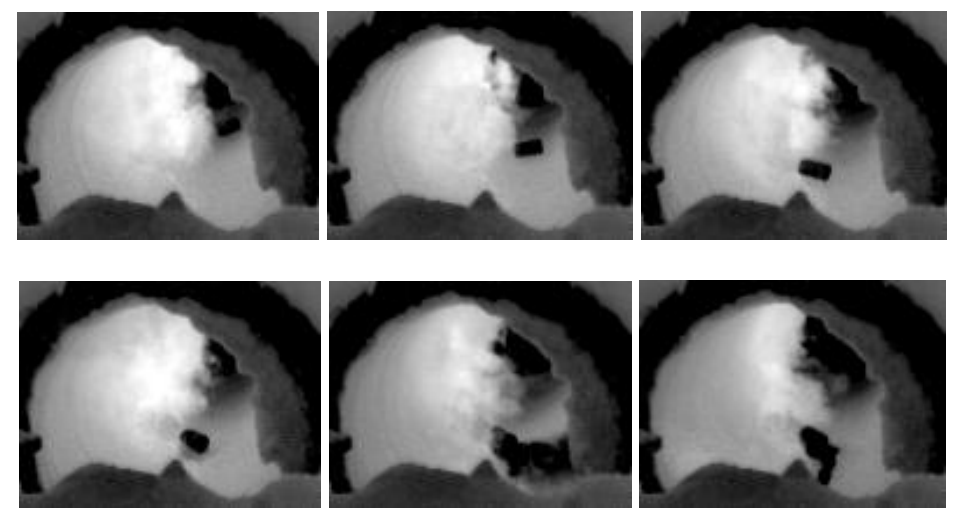

Fig. 12. Image Sequence of barrel rolling through almost the whole kiln

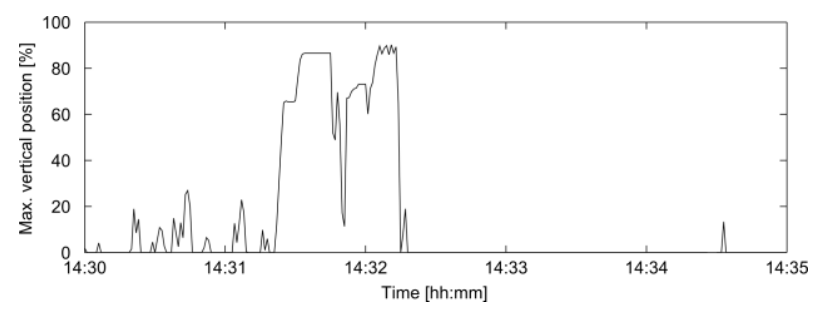

Fig. 13. Maximum vertical position of barrel

\subsubsection{Open points}

Due to occasional cleaning flushings of the burner for paste-like materials it is possible that cold paste-like material ends up in the kiln. By use of the presented method this material might also be classified as a barrel. At a lower camera position the height of the barrels could be considered as an additional plausibility criterion. Moreover, a better image resolution in the ROI could enable the integration of prior knowledge about the barrel's shape in the segmentation step.

Furthermore, cakings in the camera view field constrain the observable image area and can lead to false segmentations as well as missed barrels. By building a special protective shield to reroute the slag this drawback can be obviated.

If there is a poor sight into the kiln e.g. because of high particle concentrations in the gas phase the method cannot be employed robustly. 


\section{Conclusions}

Based on the available image data it can be shown that a camera-based analysis of barrels in rotary kilns for waste incineration is possible. By observing the extracted feature of the barrel's size a deflagration can be detected before the associated flue gases reach the afterburner chamber. Therefore it is feasible to adapt the process control accordingly in time. The feature of the vertical position of the barrel can be employed as a detector for barrels that are rolling through the entire kiln without burning out completely.

Future works comprise the adjustment of the camera system (optical characteristic, mounting position) for a continuous operation. Afterwards adaptations of the algorithm as well as improvements regarding robustness will be performed.

\section{REFERENCES}

[1] Boateng A.A., " Rotary Kilns: Transport Phenomena and Transport Processes", vol. 1, 2008.

[2] Matthes J., Waibel P., Keller H.B., "A new Infrared Camera-based Technology for the Optimization of the Waelz Process for Zinc Recycling". Minerals Engineering, vol. 24, pp. 944-949, 2011.

[3] CURRENTA GmbH, "Incineration Plants: Hazardous Waste Incineration, Wastewater Incineration, Sewage Sludge Incineration", 2010 